\title{
MEASUREMENT OF THE DYNAMICS OF THE SHEAVE BLOCK OF SHIPYARD CRANE WITH 1000 T LIFTING CAPACITY FOR VERIFICATION OF THE LABORATORY STAND
}

\author{
Piotr Szymak ${ }^{11}$, Pawel Piskur'1), \\ Jacek A. Goszczyński ${ }^{1}$, Tomislav Batur ${ }^{2}$ \\ ${ }^{1)}$ FUD Technologies Sp. z o.o. (Poland) \\ ${ }^{2)}$ University of Split (Croatia)
}

\begin{abstract}
The paper includes partial results of the development project entitled "The first Polish gantry crane with high lifting capacity for the shipbuilding industry adapted to work in difficult wind conditions - development and demonstration" (no POIR.01.02.00-00-0017/18). Due to the requirements of working in challenging wind conditions, the crane will be equipped with the sheave block with a hook operating automatically. To resolve this problem, mathematical and then physical models of the innovative crane are required. Therefore, to obtain reliable models, the measurement of the dynamics of the sheave block of crane with high lifting capacity was carried out.

At the beginning of the paper, the conditions and used measurement stand are included. Then, the time and frequency analysis of the received measurement results are described. Moreover, the determination of the crane parameters is also presented. The analysis results will be used in further research to tune both the simulation and the physical models of the crane with an automatic hook, which are being developed.

Keywords: measurement; dynamics; gantry crane; load block; time analysis; frequency analysis
\end{abstract}

\section{Introduction}

The building process of large steel structures, especially ships, requires the use of large lifting devices. One such device is a gantry crane, quite often used in the shipbuilding industry to assemble individual sections of a ship. These cranes' operation requires the use of a team of people directly as operators and supporting the crane hook of the load block with the transported load to hoisting it. Unfortunately, the technical inspection regulations prohibit people's presence close to the load block in the event of strong winds up to $20 \mathrm{~m} / \mathrm{s}$. Moreover, operation in the area close to the large load block is hazardous (Milazzo et al. 2021). 
Therefore, there is a need for automation of connecting the crane block with the load. There is a lack of commercial solutions in this field, considering large gantry cranes.

Therefore, Polish development project no POIR.01.02.00-00-0017/18 called Goliat devoted to such problems is carried out currently. The project's object is to create and demonstrate the first Polish, innovative gantry crane that can handle huge, irregularly-shaped pieces of construction produced and transported in shipyards, ports, and offshore component factories. Implementation of the Project will contribute to shipbuilding, port industry, and other heavy industry by focusing on the automation of technological processes and reducing weather impact (wind force) on work efficiency and cargo throughput. It will reduce the cost of production and increase the competitiveness of the Polish shipbuilding industry.

Automatic connecting and consequent hoisting of transported load demands the development of two leading technologies: precise crane hook positioning relative to lug mounted to the load and robust control algorithms of automatic hook. Considering the relative positioning, two main solutions can be found. The first is connected with using Attitude Heading Reference System (AHRS) based on MicroElectroMechanical System (MEMS) technology with ZigBee communication (Gao et al. 2014). The second one is Surface Acoustic Wave Radio Frequency IDentification (SAW RFID) localization (Lyu et al. 2019). Considering control algorithms, different solutions may be used, e.g., nonlinear coupling anti-swing control method (Shi et al. 2019), a controller designed based on uniformly ultimately bounded (UUB) theory (Park et al. 2021), classical PID, or other nonlinear control strategies.

In the Goliat project, the positioning method based on video image processing is proposed. Moreover, linear and nonlinear controllers are currently tuned using numerical and then physical models of gantry cranes with the automatic hook. To validate these models, the measurement of a large gantry crane is needed, which is the main subject of this paper.

In the next section, the measurement conditions and stand are presented. Then, the different analyses of the obtained results are included. At the end, the conclusions with the future research schedule are formulated.

\section{Measurement of the load block dynamics}

\section{Object}

Measurements of the block dynamics were carried out in real conditions using one of the crane load blocks with a $1000 \mathrm{t}$ lifting capacity, located at the Crist S.A. shipyard (Figure 1). 


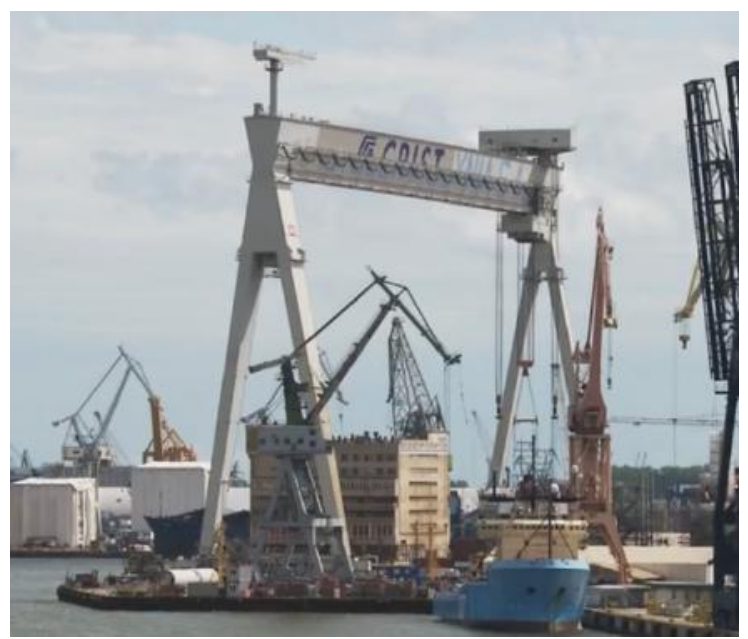

Figure 1. Gantry crane with a $1000 \mathrm{t}$ lifting capacity in Crist S.A. shipyard ${ }^{1)}$

In Figure 2, the examined load block with the measuring device is visualized.

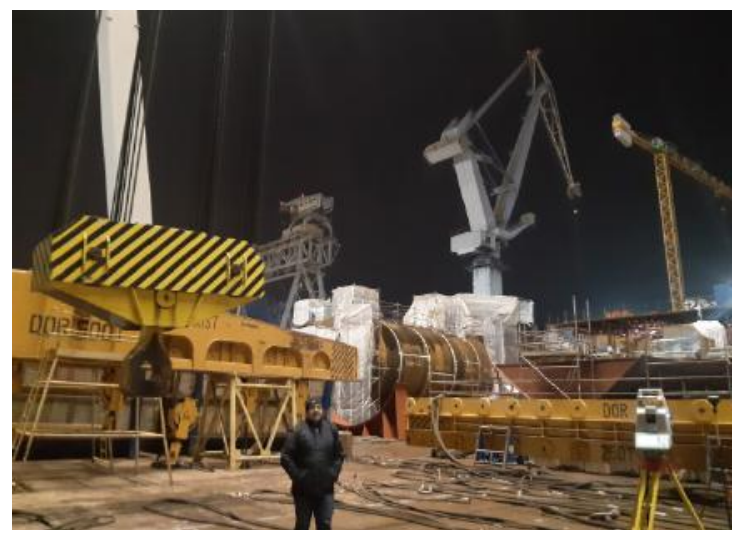

Figure 2. View of the load block and total station theodolite during measurements at the Crist S.A. shipyard

\section{Measuring devices}

Measurements were made using the following measuring devices:

1. Leica Nova TS6 Total Station Theodolite (TST),

2. Leica GS18T GNSS receiver with CS20 controller.

Using the devices mentioned above with appropriate geodetic alignment algorithms allowed us to measure the hook position in the space with a few 
millimeters accuracy, sufficient for the load block dynamics measurement. Details of the measurements and the parameters of the used devices are included in (Świerczyński \& Marchel 2020).

\section{Conditions}

Dynamics measurements were carried out under the influence of wind speed approx. $16 \mathrm{~m} / \mathrm{s}$ for the block in the upper position and $1.5-3 \mathrm{~m} / \mathrm{s}$ in the lower one.

The block was lowered into the working zone used for its conversion, sheltered from the wind up to a height of 10 to $15 \mathrm{~m}$, depending on the direction. Hence the minimized influence of the wind was recorded in the lower part of the block position. The upper position of the block was observed at the height of approx. $70 \mathrm{~m}$, and the lower one at the height of 2 to $3 \mathrm{~m}$ from the ground, depending on the operator's reaction.

The block dynamics measurements presented below were converted to the local system illustrated in Figure 3.

The block dynamics measurements were carried out during the following motions:

1. From the lower to the upper position with a nominal speed of $12 \mathrm{~m} / \mathrm{s}$ (high speed).

2. From the top to the bottom position with a high speed.

3 . From the lower position to a height of over $40 \mathrm{~m}$ with a high speed.

4. From a height of over $40 \mathrm{~m}$ to the lower position with a nominal speed of 4 $\mathrm{m} / \mathrm{s}$ (medium speed),

5. From the lower position to a height of more than $20 \mathrm{~m}$ with a high speed.

6 . From over $20 \mathrm{~m}$ high to over $10 \mathrm{~m}$ high with a nominal speed of $1 \mathrm{~m} / \mathrm{s}$ (low speed), then return to the down position with high speed.

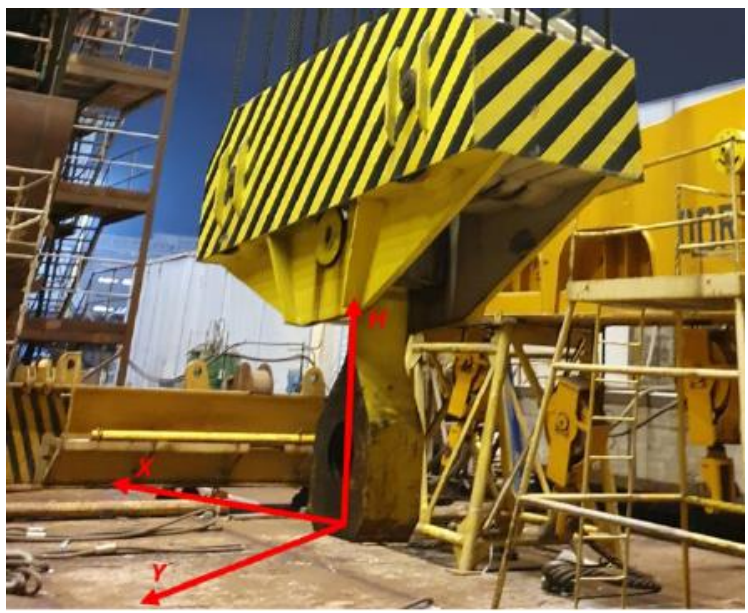

Figure 3. The local coordinate system for block dynamics measurement 
Important from the point of view of the hook's automatic operation is the dynamics of the block during its lowering, particularly with low speed, called maneuvering in the range of lower positions from a few to several meters. Therefore, further measurements analyses were conducted for the block motions from higher to lower heights.

\section{Analyses of the results}

\section{Time Analysis}

Figure 4 shows the changes in the relative height $h$, the coordinates $x$ and $y$, and the distance $\mathrm{d}$, calculated as the distance from the initial position on the horizontal plane. The charts show the parameters' changes for lowering the load block from the upper to the lower position. Due to the significant increase in the measurement time at medium and low speeds and the related downtime in the yard's production activities, the block's upper position for medium speed was set at $40 \mathrm{~m}$ and for low speed at $20 \mathrm{~m}$. As a result, a comparable dynamics measurement time was obtained (Figure 4.a). It should be mentioned that the received measurement time covers several dozen periods of the observed basic vibrations of the block. Moreover, it should be noted that the starting point as mentioned above was determined as the mean values of the coordinates $\mathrm{x}$ and $\mathrm{y}$ in the upper block position, i.e., all measurements were visualized with respect to the same initial block location.

(a)

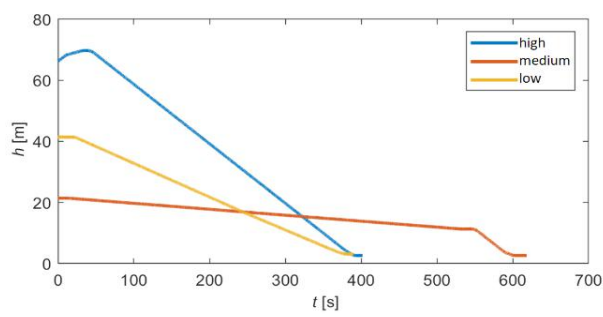

(c)

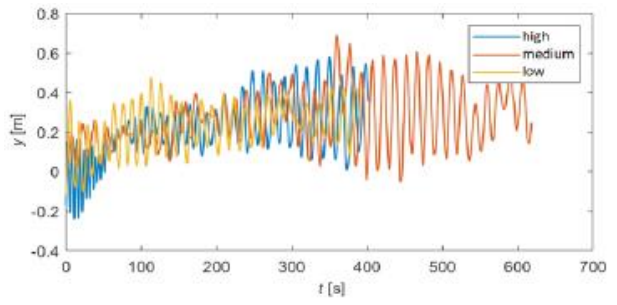

(b)

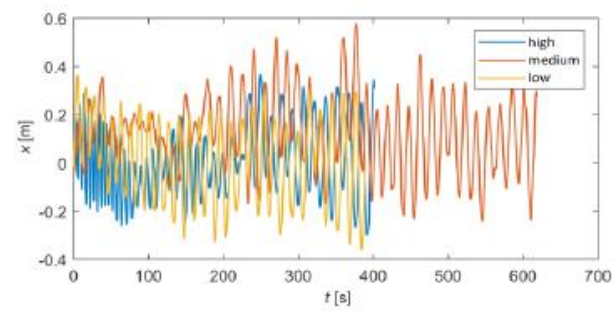

(d)

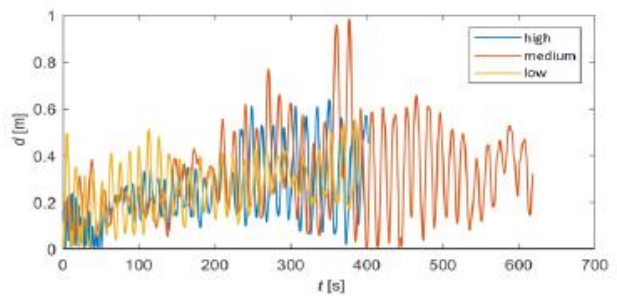

Figure 4. Comparison of the dynamics of the load block during its lowering for different speeds of motion: changes in height h(a), coordinates $\mathrm{x}$ (b) y (c), distance d (d) 
Based on the visualized data (Figure 4), the mean displacement in the OX axis close to zero, and the average displacement in the $0 \mathrm{Y}$ axis at the level of $0.3 \mathrm{~m}$ may be estimated. The largest oscillations (amplitudes) can be observed for the low speed, while the smallest for the high speed in its initial course. It probably results from the assumed initial position, i.e., at different speeds, the measurement starts from the different heights (different rope lengths). Moreover, the smallest oscillation amplitudes are observed at the beginning of the measurement, i.e., when the block is affected by additional acceleration associated with the downward movement's beginning.

The following Figure 5 presents a comparison of the block trajectories on the horizontal surface for different speeds of motion when reaching the lower position. It can be seen that the block performs repetitive movements with a fixed trajectory. In this case, there should be no problem with predicting the block position, which will allow for automatic connection of the block with the lug.

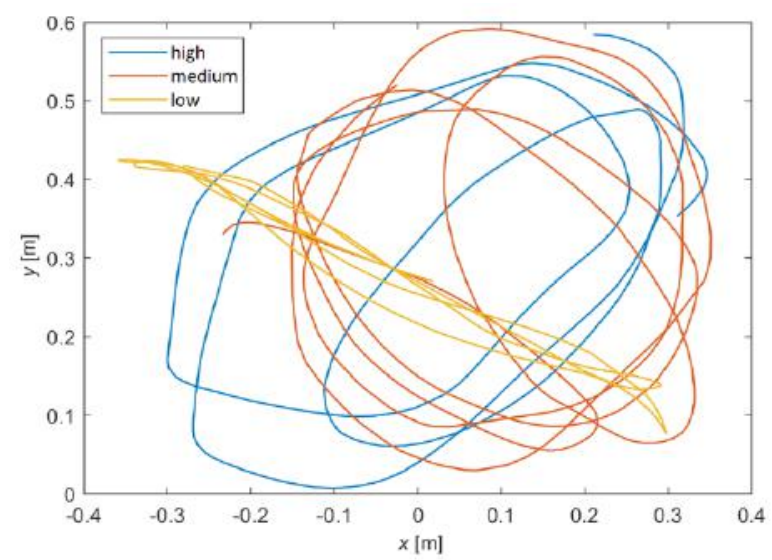

Figure 5. Comparison of the block trajectories for different speeds when reaching the lower position

In Table 1, the statistical parameters were collected for the changes of the coordinates $\mathrm{x}$ and $\mathrm{y}$ when the load block was lowered with different speeds. Overall, the obtained values were similar in the horizontal plane for different speeds. The highest values of the mean deviation of the $\mathrm{x}$ and $\mathrm{y}$ coordinates were observed for the lowest speed of lowering the block.

Apart from the mean position deviation in the horizontal plane, one can observe the oscillations of coordinates $\mathrm{x}$ and $\mathrm{y}$ with quite large amplitude values. Therefore, it is crucial to perform a frequency analysis of the obtained results of block dynamics measurements. The results of this analysis are included in the following subsection. 
Table 1. Comparison of statistical parameters for changes of the coordinates $x$ and $\mathrm{y}$ when the block is lowered with different speeds

\begin{tabular}{|l|l|l|l|}
\hline Parameters & $\begin{array}{l}\text { High } \\
\text { speed }\end{array}$ & $\begin{array}{l}\text { Medium } \\
\text { speed }\end{array}$ & $\begin{array}{l}\text { Low } \\
\text { speed }\end{array}$ \\
\hline $\boldsymbol{x}_{\text {ś }}[\mathrm{m}]$ & 0.016 & 0.012 & 0.127 \\
\hline $\boldsymbol{x}_{\min }[\mathrm{m}]$ & -0.299 & -0.359 & -0.242 \\
\hline $\boldsymbol{x}_{\max }[\mathrm{m}]$ & 0.369 & 0.364 & 0.580 \\
\hline $\boldsymbol{y}_{\text {sr }}[\mathrm{m}]$ & 0.226 & 0.231 & 0.269 \\
\hline $\boldsymbol{y}_{\min }[\mathrm{m}]$ & -0.240 & -0.117 & -0.051 \\
\hline $\boldsymbol{y}_{\max }[\mathrm{m}]$ & 0.584 & 0.4775 & 0.693 \\
\hline
\end{tabular}

\section{Frequency Analysis}

In Figure 6, the spectra of the coordinates $\mathrm{x}$ and $\mathrm{y}$ for different speeds of lowering the block were presented. Based on the signal spectra, two essential components can be observed: a constant at $0 \mathrm{~Hz}$ and the first harmonic at a frequency of approx. $0.065 \mathrm{~Hz}$ with an amplitude comparable (high speed) and almost twice as high (medium and low speeds) with the amplitude of the constant component. The relatively large amplitude of the harmonic component forces the need to include it in the control algorithm. It can be additionally observed a harmonic component at the frequency of $0.19 \mathrm{~Hz}$ for high speed but with an amplitude almost four times smaller than the amplitude of the constant and fundamental harmonic components.

(a)

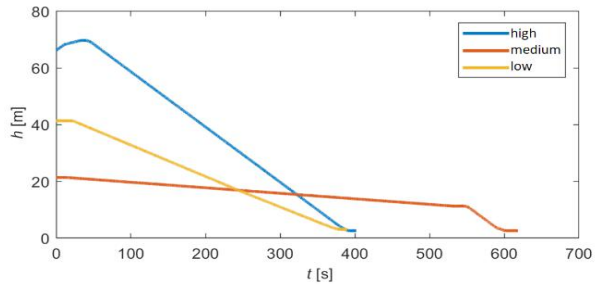

(c)

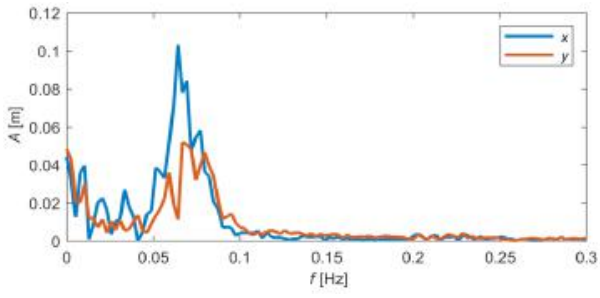

(b)

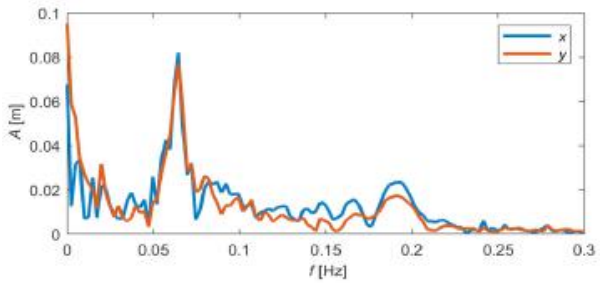

(d)

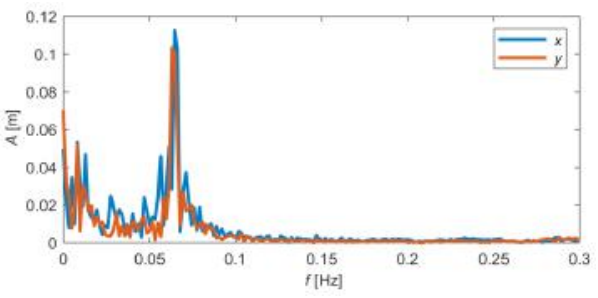

Figure 6. Comparison of the dynamics of the load block during its lowering: changes in height $h(a)$, spectra of coordinates for high (b), medium (c), and low (d) speeds 
For all speeds of motion, the same frequency of the first harmonic is obtained, which allows for the conclusion that it is not dependent on the speed of movement, but is characteristic of the gantry structure. To explain the origin of this component, the FFT analysis was performed for various relative heights. The analysis results are presented in Figure 7, and the determined dominant frequencies are visualized in Table 2.
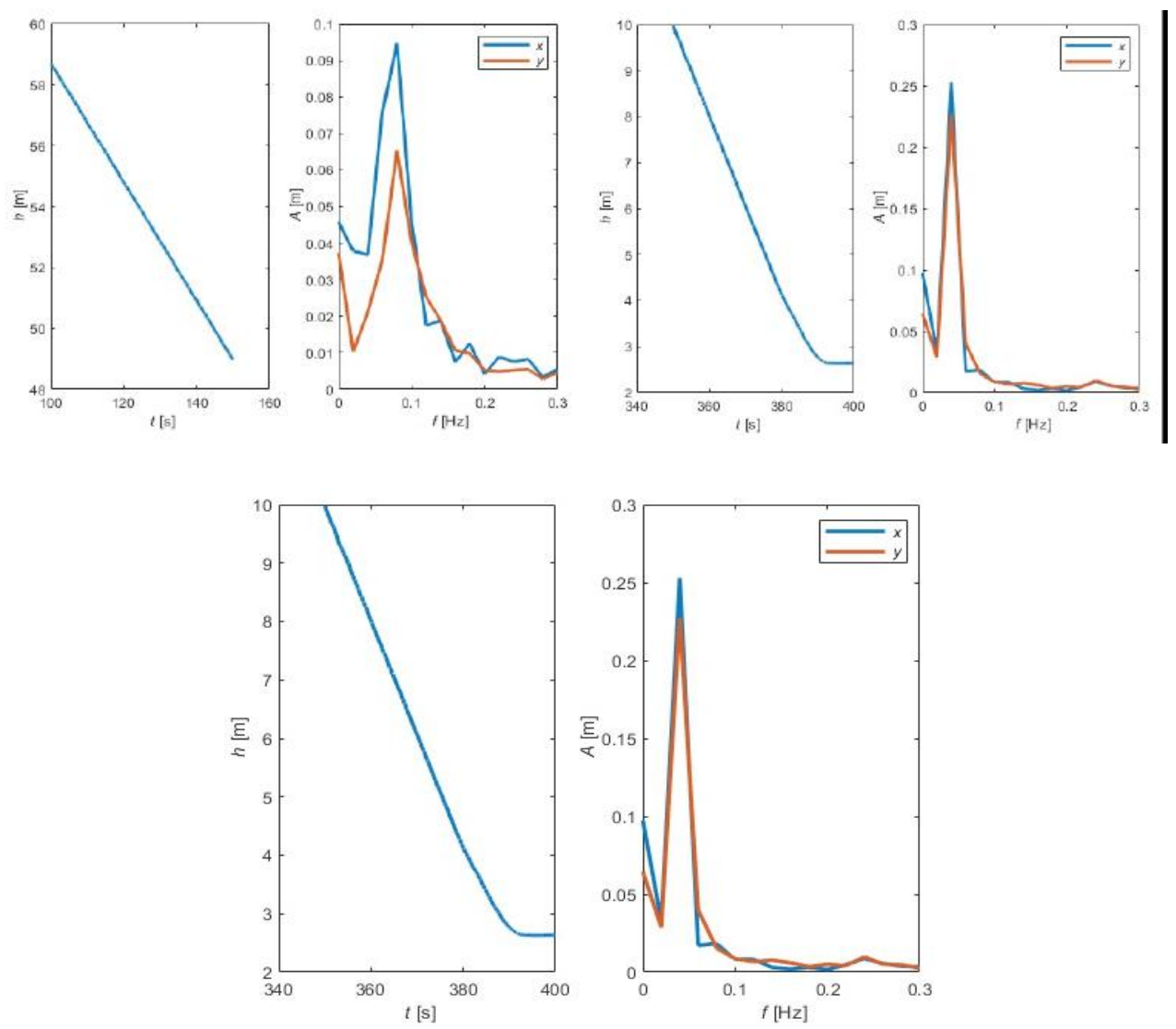

Figure 7. Spectra of the coordinates $x$ and $y$ of the load block for the selected ranges of the height for motion with the high speed

It can be noticed that as the relative height decreases, i.e., the length of the crane ropes increases, the frequency of the harmonic component decreases, and its amplitude increases. This behavior is consistent with the mathematical pendulum theory for small angles of the vertical pendulum deviation. 
Table 2. Dominant frequencies for the successive ranges of the height of the load block for the motion with the high speed

\begin{tabular}{|l|l|l|l|l|}
\hline $\boldsymbol{h}_{\min } \ldots \boldsymbol{h}_{\max }[\mathrm{m}]$ & $\mathbf{6 6 . . . 7 0}$ & $\mathbf{5 9 \ldots 6 8}$ & $\mathbf{4 9} \ldots 59$ & $39 \ldots 49$ \\
\hline $\begin{array}{l}\boldsymbol{f}_{d} \\
{[\mathrm{~Hz}]}\end{array}$ & 0.185 & 0.12 & 0.08 & 0.07 \\
\hline $\boldsymbol{h}_{\min } \ldots \boldsymbol{h}_{\max }[\mathrm{m}]$ & $29 \ldots 39$ & $20 \ldots 29$ & $20 \ldots 10$ & - \\
\hline $\begin{array}{l}\boldsymbol{f}_{d} \\
{[\mathrm{~Hz}]}\end{array}$ & 0.06 & 0.05 & 0.04 & - \\
\hline
\end{tabular}

\section{Identification of the Motion Parameters}

The block was lowered with three different nominal speeds, i.e., high $12 \mathrm{~m} / \mathrm{min}$, medium $4 \mathrm{~m} / \mathrm{min}$, and low $1 \mathrm{~m} / \mathrm{min}$. The obtained measurements allow us to determine the actual speed values. Figure 8 illustrates the changes in height and the corresponding "raw" and filtered changes in the speed of movement in the vertical axis for the speeds mentioned above.

Table 3 presents the obtained results of the speed of movement. They were calculated as the arithmetic mean for measurements on rectilinear sections of height changes, e.g., for high speed from 50 to $350 \mathrm{~s}$ of measurements, etc. The calculated values of the speed of movement in the vertical axis do not take into account the starting and braking moments of the block.

Table 3 shows that the medium speed is practically equal to the speed value of approx. $7 \mathrm{~m} / \mathrm{min}$, i.e., it is almost twice as high as the nominal value. Also, the low speed is higher, but only by about $20 \%$ of the nominal value.

(a)

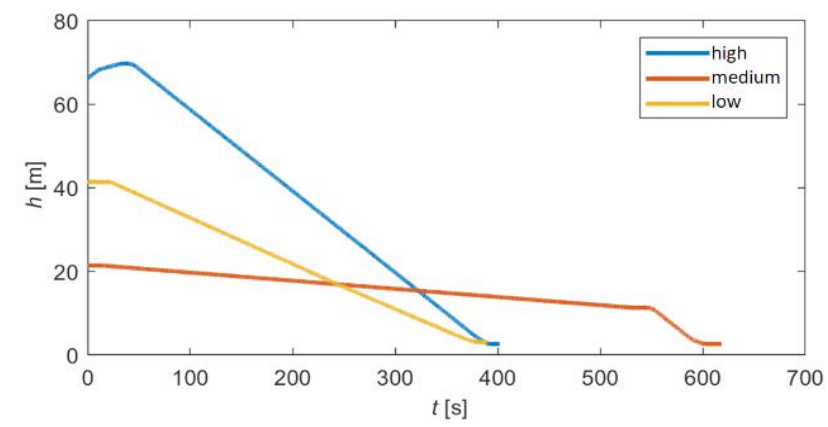


(b)

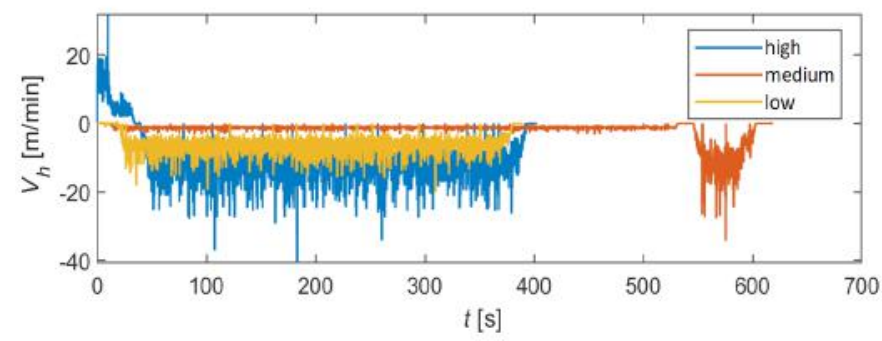

(c)

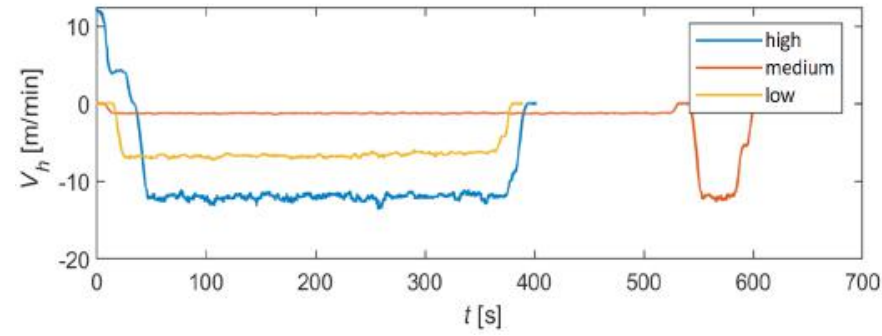

Figure 8. Comparison of the dynamics of the block during its lowering for different speeds of movement: a) changes in height $\mathrm{h}$ (a), speed of movement along the vertical axis Vh: unfiltered (b), a filtered average of 30 samples (c)

Table 3. Real speed in the vertical axis obtained for different nominal speeds of the load block movement

\begin{tabular}{|l|l|l|l|}
\hline $\begin{array}{l}\text { Nominal speed } \\
{[\mathrm{m} / \mathrm{min}]}\end{array}$ & $\mathbf{1 2}$ & $\mathbf{4}$ & $\mathbf{1}$ \\
\hline $\begin{array}{l}\text { Real speed } \\
{[\mathrm{m} / \mathrm{min}]}\end{array}$ & 12.08 & 6.731 & 1.204 \\
\hline
\end{tabular}

In the course of further analysis, the load block's acceleration and deceleration times were determined for different speeds of movement using a filtered speed Vh. Due to the irregular (oscillating) nature of speed changes, the acceleration and deceleration times were selected for the speed change range from $10 \%$ to $90 \%$ of the set speed value. The results of this analysis are presented in Table 4. Moreover, Figure 9 shows the acceleration and deceleration of the load block for three different speeds. 
As can be seen from the data collected in Table 4, the deceleration times for high and medium speeds are almost two times greater than the acceleration times. For low speed, similar values of acceleration and deceleration times were obtained. The greater deceleration times in relation to the acceleration in the vertical axis can be explained by the influence of gravity, which supports the process of starting the electric motors driving the load block.

(a)

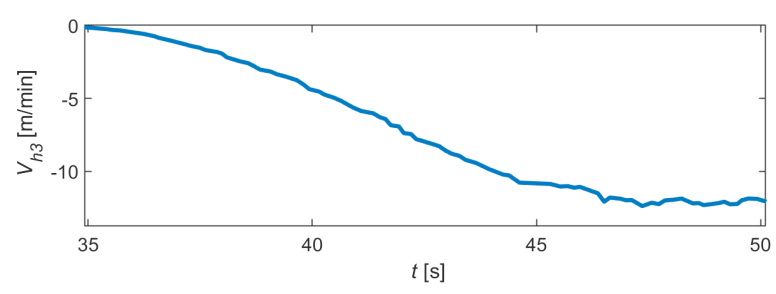

(b)

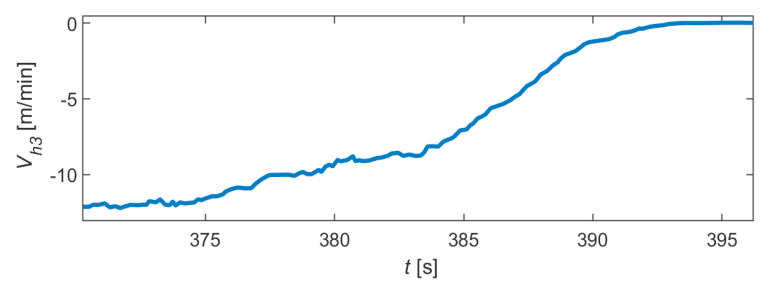

(c)

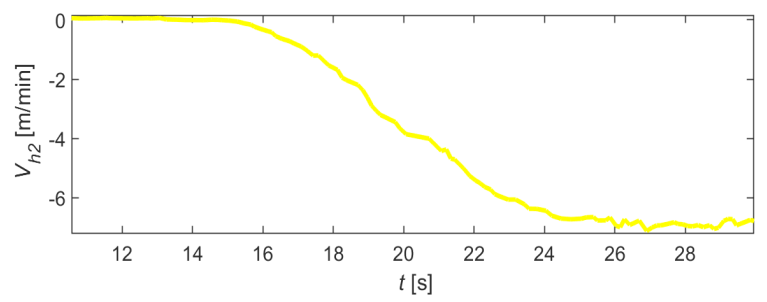

(d)

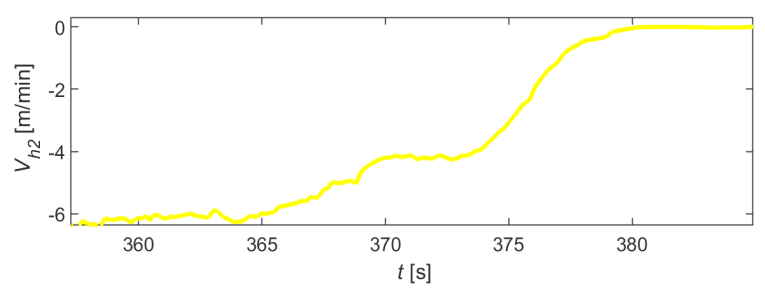


(e)

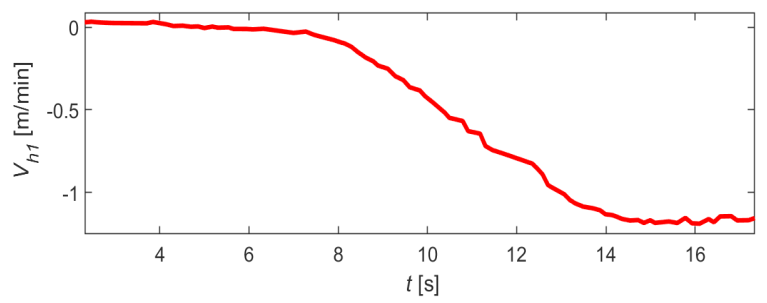

(f)

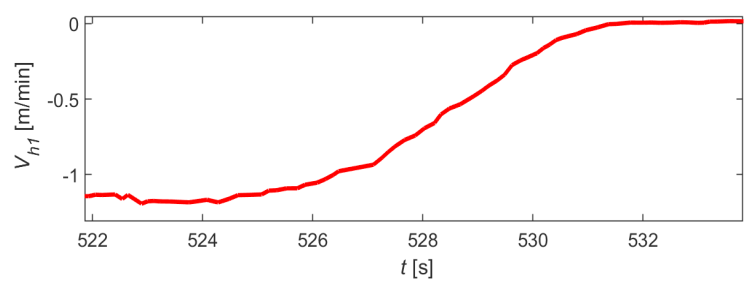

Figure 9. Speed changes in the vertical axis Vh for the moments of acceleration a) c) e) and deceleration of the block b) d) f) for high, medium, and low speed, respectively

Table 4. Acceleration and deceleration times for different block speeds

\begin{tabular}{|l|l|l|l|}
\hline & High speed & $\begin{array}{l}\text { Medium } \\
\text { speed }\end{array}$ & $\begin{array}{l}\text { Low } \\
\text { speed }\end{array}$ \\
\hline $\begin{array}{l}\text { Acceleration } \\
\text { time } \\
{[\mathrm{s}]}\end{array}$ & 7.5 & 6.2 & 5.2 \\
\hline $\begin{array}{l}\text { Deceleration } \\
\text { time } \\
{[\mathrm{s}]}\end{array}$ & 13.3 & 12.6 & 6.1 \\
\hline
\end{tabular}

\section{Conclusions}

The use of precise geodetic devices with appropriate geodetic alignment algorithms allowed for obtaining high accuracy of the block dynamics measurement (positioning errors at the level of several millimeters), which enabled the precise size of its trajectory in space during its movement.

The analyses of the obtained measurements allowed for the formulation of the following conclusions:

1. The average deviation of the block from the initial position increases with the ropes' length, and in the down position, it gets values less than $0.3 \mathrm{~m}$. 
2. Apart from the constant deflection caused by the impact of the wind, oscillations of decreasing frequency were observed on the block suspended on a longer line,

3. The above deviations from the set initial position are determined, and it is possible to predict the block position in a relatively long time perspective (slowchanging process).

The above conclusions allow for constructing an automatic hook control algorithm resistant to environmental disturbances in the form of wind with a speed of $16 \mathrm{~m} / \mathrm{s}$.

The measured block had larger dimensions and mass than the planned block in the gantry crane design, which allows us to predict that the wind's influence will be more negligible for the designed block. Despite the promising results of the dynamics measurement, it should be repeated for the designed block in various environmental conditions. It will allow us to tune the control algorithm, including the algorithm for block position prediction.

Acknowledgments. The results presented in the paper have been obtained within the development project No. POIR.01.02.00-00-0017/18 financed by the Polish National Centre of Research and Development in 2019 - 2022 years. The $1000 \mathrm{~T}$ lifting capacity gantry crane's measurement has been carried out courtesy of the Crist S.A. harbor's Owners.

\section{NOTES}

1. https://www.trojmiasto.pl/wiadomosci/Gdynia-nowy-napis-na-wielkiej-suwnicyn113767.html).

\section{REFERENCES}

Gao, B., Zhu, Z., Zhao, J., Huang, B., 2014. A Wireless Swing Angle Measurement Scheme Using Attitude Heading Reference System Sensing Units Based on Microelectromechanical Devices. Sensors 14, 22595 - 22612.

Lyu, J., Shi, R., Yang, Y., Han, T., 2019. Matching Detection of Crane Hook and Ladle Lug before Ladle Hoisting. Sensors 19, 5389.

Milazzo, M.F., Ancione, G., Consolo, G. 2021. Human Factors Modelling Approach: Application to a Safety Device Supporting Crane Operations in Major Hazard Industries. Sustainability 13, 2304.

Park, H.-C., Chakir, S., Kim, Y.-B. \& Lee, D.-H., 2021. A Robust Payload Control System Design for Offshore Cranes: Experimental Study. Electronics 10, 462. 
Piskur, P., 2021. Development and implementation of the measuring system and measurements of a block's dynamics with an automatic hook in real conditions - time analysis, partial report of project No POIR.01.02.0000-0017/18, 2021.

Shi, H., Li, G., Ma, X. \& Sun, J., 2019. Research on Nonlinear Coupling Anti-Swing Control Method of Double Pendulum Gantry Crane Based on Improved Energy. Symmetry 11, 1511.

Świerczyński, S., Marchel, Ł., 2020. Development of the concept and performance of measurements of the block's dynamics with a hook in real conditions, partial report of project No POIR.01.02.00-00-0017/18, 2020.

Szymak, P., 2021a. Development and implementation of the measuring system and measurements of a block's dynamics with an automatic hook in real conditions - frequency analysis, partial report of project No POIR.01.02.00-00-0017/18, 2021.

Szymak, P., 2021b. Processing the results of block dynamics measurements with a hook for a dynamic numerical model and a physical model in scale, partial report of project No POIR.01.02.00-00-0017/18, 2021.

$\triangle$ Piotr Szymak

https://orcid.org/0000-0002-4714-6192

FUD Technologies Sp. z o.o.

Gdynia, Poland

E-mail: piotr.szymak@fudtech.pl

$\triangle$ Pawel Piskur

https://orcid.org/0000-0002-8823-4316

FUD Technologies Sp. z o.o.

Gdynia, Poland

E-mail: paweł.piskur@fudtech.pl

$\triangle$ Jacek A. Goszczyński

FUD Technologies Sp. z o.o.

Gdynia, Poland

E-mail: jacek.goszczynski@fudtech.pl

$\triangle$ Tomislav Batur

Faculty of Maritime Studies

University of Split

Split, Croatia

E-mail: baturmeister@gmail.com 